\title{
Diversidade taxonômica e distribuição geográfica das solanáceas que ocorrem no Sudeste Brasileiro (Acnistus, Athenaea, Aureliana, Brunfelsia e Cyphomandra)
}

\author{
Lucia d'Ávila. Freire de Carvalho ${ }^{1}$ \\ Lucio Heron P. Costa ${ }^{2}$ \\ Aline Castellar Duarte ${ }^{3}$
}

\begin{abstract}
RESUMO
Os gêneros registrados para a região Sudeste (Acnistus, Athenaea, Aureliana, Brunfelsia e Cyphomandra) são analisados com base na documentação bibliográfica e de herbário. Para interpretar os diversos aspectos do hábito, habitat e distribuição geográfica, foram elaboradas tabelas específicas. Os resultados identificam a região como um provável Centro de Diversidade específica. É acrescentada uma análise da situação dos 39 táxons quanto à preservação, citando as Unidades de Conservação onde estão localizados. Uma chave analítica é desenvolvida para o reconhecimento dos táxons visando auxiliar taxonomistas e estudiosos da florística.
\end{abstract}

Palavras-chave: Solanaceae, Diversidade, Distribuição Geográfica, Floresta Atlântica, Sudeste, Brasil.

\begin{abstract}
Using the literatura and herbarium material, the genera recorded for the Southeast region (Acnistus, Athenaea, Aureliana, Brunfelsia e Cyphomandra) are analyzed. Species tables describe the various aspects of life form, habitat and geographical distribution and identify the region as a centre of diversity. It is included an analysis of the preservation status of the taxons, listing conservation units where they are found. An analytic key was developed for the recognition of the taxons, aiming to help taxonomists and floristic researchers.

Keywords: Solanaceae, Diversity, Geographical distribution, Atlantic forest, Southeast, Brazil.
\end{abstract}

\section{INTRODUÇÃO}

Para Hunziker (1979) a maior riqueza de espécies da família Solanácea pode ser encontrada na América do Sul. Purdie, Symon e Helgi (1982) reconhecem cerca de 90 gêneros e estimam em 2600 espécies de solanáceas, com ampla distribuição geográfica nas regiões tropicais e temperadas, a maioria nativa das Américas do Sul e Central. Em 1991, D’Arcy reconheceu para a família 96 gêneros com aproximadamente 2297 espécies e de acordo com os colegas solanólogos afirma que a maior diversidade está concentrada na América do Sul, onde cerca de 50 gêneros são endêmicos.

Para o Brasil não existe nenhuma estimativa da riqueza de espécies, a exceção da monografia de Sendtner (1846) publicada na Flora brasiliensis de Martius.

Carvalho, Costa e Castellar (2001, no prelo) divulgam a riqueza de espécies encontrada para a região sudeste, através de uma listagem, indicando 17 gêneros representados por 254 espécies, mais os táxons infraespecíficos.

\footnotetext{
${ }^{1}$ Instituto de Pesquisas Jardim Botânico do Rio de Janeiro - Programa Diversidade Taxonômica - CNPq.

${ }^{2}$ Biologo do Herbário Bradeanum

${ }^{3}$ Bolsista do Jardim Botânico do Rio de Janeiro/CNPq
} 
A partir desta listagem elaborada, estabeleceu-se um grupo de cinco gêneros com revisão taxonômica (Acnistus, Athenaea, Aureliana, Brunfelsia e Cyphomandra), documentados por 39 táxons (tabela 1), para desenvolver os estudos sobre os aspectos fitogeográficos, e procurar identificar o centro de diversidade.

Os temas abordados, relativos ao hábito das plantas, meio ambiente em que se desenvolvem e a distribuição geográfica, associados a leitura dos mapas político e da vegetação (Góes Filho, 1992) objetiva fornecer conhecimentos sobre a biologia dessas espécies.

Pretende-se, também, com estes estudos,oferecer subsídios relevantes sobre a participação das Solanáceas na composição florística da região que ocupa área representativa entre os trópicos, delimitada pela costa atlântica.

\section{MATERIAL E METODOS}

A divulgação de listagens, flórulas estaduais e locais, revisões taxonômicas, consultas aos trabalhos clássicos e das coleções botânicas herborizadas (ALCB, ASE, FLOR, FZB, GUA, HB, HBR, ICN, MBM, PACA, $\mathrm{R}, \mathrm{RB}, \mathrm{SP}, \mathrm{UEC}$ ), tornou possível a elaboração da listagem de táxons representados na região sudeste que serviu de base para este estudo (Carvalho, Costa e Castellar, 2001, no prelo). As siglas dos herbários citados estão de acordo com Holmgren et al. (1990).

Os dados referentes as formas de vida, habitat, distribuição geográfica e unidades de conservação de cada uma das espécies, foram extraídos das etiquetas das coleções herborizadas acrescentando-se a interpretação dos resultados da pesquisa bibliográfica.

A chave analítica para identificar os táxons foi elaborada com base nas revisões taxonômicas dos gêneros estudados.

O tipo de vegetação da região foi classificada segundo o sistema elaborado por Veloso et al. (1991).
A categoria das unidades de conservação seguem os conceitos estabelecidos por Buck et al. (1995).

A transferência do gênero Cyphomandra para o gênero Solanum (Bohs, 1995) não foi considerada neste levantamento.

\section{DOCUMENTAÇÃO DOS TÁXONS EXAMINADOS}

Acnistus Schott

Acnistus arborescens (L.) Schlecht., Linnaea 7: 67. 1832. Localidade Tipo: América Meridional. Minas Gerais: prox. Rio Paraíba, M. Nee 3441 (18.X.1970) RB, floresta; Rio de Janeiro: Grumari, Araújo 546 (16.XI.1973) RB, restinga; São Paulo: Miracatu prox. Rio São Lourenço, Mattos 9133 (28.IX.1961) SP, capoeira;

\section{Athenaea Sendtn.}

Athenaea anonacea Sendtn. in Mart. Fl. Bras. 10: 137. 1846. Localidade Tipo: Brasil austral. Minas Gerais, Claussen (1834-43) BM; Rio de Janeiro: Munic. de Nova Friburgo, Macaé de cima, I. de Araújo 36 (05.VII.1989) RB, floresta;

Athenaea cuspidata Witas., Denkschr. Math. - Naturw. 79: 318. 1931. Localidade Tipo: São Paulo. Espírito Santo: Domingos Martins, Peixoto 474 (19.III.1975) RB, floresta; São Paulo: Caraguatatuba, R. F., Mattos 13740 (24.V.1966) SP;

Athenaea martiana Sendtn. in Mart. Fl. Bras. 10: 136. 1846. Localidade Tipo: Minas Gerais, Sobradinho; Rio de Janeiro, Paraibuna. Minas Gerais: Chapeco d'Uva, Gardner 5047 (X.1840) BM; Espírito Santo: Castelo, Brade 19222 (09.VIII.1948) RB, floresta;

Athenaea micrantha Sendtn. in Mart. Fl. Bras. 10: 135. 1846. Localidade tipo: Minas 
Diversidade taxonômica e distribuição geográfica das solanáceas que ocorrem no Sudeste

Brasileiro (Acnistus, Athenaea, Aureliana, Brunfelsia e Cyphomandra)

Gerais, Viçosa. Minas Gerais: Serra da Cayana, Schwake s/n. (3.VII.1888) R; Espírito Santo: Morro Dantas Lanna Sobrinho 1008(8.VIII.1965) GUA; Rio de Janeiro: P.N. Itatiaia, Sucre 5809 (17.VIII.1969) RB, floresta; São Paulo: São José dos Campos, Löfgren 276 (12.III.1909) RB.

Athenaea pereirae Barboza et A.T. Hunz., Bol. Soc. Argent. Bot. 26(1-2): 100. 1989. Localidade tipo: Rio de Janeiro, Itatiaia. Minas Gerais: Serra do Espinhaço, R.F. de Ibitipoca, Hunziker 25203 (19.IV.1986) CORD, floresta; Rio de Janeiro: P.N. Itatiaia, Pereira 86 (VII.1953) RB, floresta.

Athenaea picta (Mart.) Sendtn. in Mart. Fl. Bras. 10: 134. 1846. Localidade tipo: Rio de Janeiro, Serra do Mar. Minas Gerais: Serra de Ibitipoca, Pires 25324 (29.VI.1991) RB, floresta; Espírito Santo: Santa Maria Madalena, Brade 18358 (23.V.1946) RB, floresta; Rio de Janeiro: Sumaré, Fonseca 241 (22.VII.1977) RB, floresta; São Paulo: Jundiaí, Serra do Japi, Leitão Fo 3220 (8.X.1978) UEC, floresta.

Athenaea pogogena (Moric.) Sendtn. in Mart. Fl. Bras. 10: 134. 1846. Localidade tipo: Bahia. Minas Gerais: Ouro Preto, Badini s/n. (19.VIII.1978) RB; São Paulo: Ubatuba, Viegas 3508 (14.II.1939) SP, floresta.

Aureliana Sendtn.

Aureliana brasiliana (A.T. Hunz.) Barboza et A.T. Hunz., Darwiniana 30(1-4): 105. 1991. Localidade tipo: São Paulo, Pindamonhangaba. Rio de Janeiro: P.N. Itatiaia, Brade 12748 (IX.33) RB, floresta; São Paulo: Ilha de São Sebastião, Mattos 15712 (27.XII.71) SP, floresta.

Aureliana darcyi Carvalho \& Bovini, Novon 5(3): 257. 1995. Localidade tipo: Rio de Janeiro, Paraty. Rio Janeiro: Paraty, Ilha das Almas, Almeida e Kurtz s.n. (07.III.1989) RB, floresta.
Aureliana fasciculata (Vell.) Sendtn. var. fasciculata in Mart. Fl. Bras. 10: 140. 1846. Localidade tipo: Rio de Janeiro. Minas Gerais: Cambuquira, Mello Barreto 7828 (25.XII.35) HB, floresta; Espírito Santo: Aracruz, Araújo et al. 241 (15.VII.73) RB, restinga; Rio de Janeiro: P.N. Itatiaia, E.Pereira et al. 84 (21.VII.53) RB, floresta; São Paulo: Itapetininga, Loefgren 325 (06.XI.87) SP.

Aureliana fasciculata var. longifolia (Sendtn.) A.T. Hunz. et Barboza, Darwiniana 30(1-4): 104. 1991. Localidade tipo: Bahia, Felisbertia; Minas Gerais. Rio de Janeiro: Munic. de Paratí, Laranjeiras, APA Cairuçu, V.L.G.Klein 956 (18.X.1990) RB, floresta; São Paulo: Cananéia, Ilha do Cardoso, Morro da Captação, Rio Pereque, Fiuza de Melo 504 (17.VIII.1983) RB, floresta.

Aureliana fasciculata var. tomentella (Sendtn.) Barboza et A.T. Hunz., Darwiniana 30(1-4): 103. 1991. Localidade tipo: Rio de Janeiro, Mangaratiba. Minas Gerais: Juiz de Fora, Brade 14102; Rio de Janeiro: Angra dos Reis, Kuhlmann 2660 (21.III.1951) SP; São Paulo: Ubatuba, Jouvin 503 (17.IV.1979) RB.

Aureliana tomentosa Sendtn. in Mart. Fl. Bras. 10: 140. 1846. Localidade tipo: Brasil austral. São Paulo: P.E. Fontes do Ipiranga, Mello 275 (25.XI.1981) SP, floresta.

Aureliana velutina Sendtn. in Mart. Fl. Bras. 10: 140. 1846. Localidade tipo: GO, Santa Cruz de Dona Tereza. Minas Gerais: Ouro Preto, Ronhel 272 (14.X.1996) OUPR, floresta.

Aureliana wettsteiniana (Witas.) A.T. Hunz. et Barboza, Darwiniana 30(1-4): 107. 1991. Localidade tipo: São Paulo, prox. a Santos. São Paulo: Serra de Paranapiacaba, P.E. Cardoso Botelho, Hoehne 6193 (18.IV.1967) SP, floresta. 
Brunfelsia (Plowman) L.

Brunfelsia bonodora (Vell.) Macbr., Field Mus. Nat. Hist. Chicago Bot. Sér. 13, 5B, (1): 152. 1962. Localidade tipo: Rio de Janeiro, Serra da Estrela. Rio de Janeiro: Serra da Estrela, Brade 18643 (29.X.1946) RB, floresta.

Brunfelsia brasiliensis (Spreng.) K.B. Smith \& Downs ssp. brasiliensis in Reitz, Fl. Ilustr. Catar. Sola: 303. 1966. Localidade tipo: Brasil meridional. Minas Gerais: P.E. do Ibitipoca, Andrade 1066 (11.XI.1987) RB, floresta; Espírito Santo: Conceição do Castelo, Hatschbach 48641 (5.XII.1984) MBM, floresta; Rio de Janeiro: Pedra da Gávea, Valente 37 (7.05.1973) RB, floresta; São Paulo: R.F. de Campos do Jordão, Davis 3018 (29.IX.1976) UEC, floresta.

Brunfelsia brasiliensis ssp. macrocalyx (Dus.) Plowman, Field Mus. Nat. Hist. Chicago Bot. n.s. 39:66.1998. Localidade tipo: Rio de Janeiro, Itatiaia. Minas Gerais: Lavras, Leitão Fo 11816 (9.XII.1910) UEC, cerrado; Rio de Janeiro: P.N. Itatiaia, Plowman 2891 (29.V.1969) RB, floresta; São Paulo: P.N. Serra da Bocaina, Brade 20953 (12.V.1951) SP, floresta.

Brunfelsia clandestina Plowman, Fieldiana, Bot. n.s. 8: 11. 1981. Localidade tipo: Bahia, Itapebi. Espírito Santo: R.F. de Linhares, Martinelli 9762 (31.X.1983) RB, floresta.

Brunfelsia cuneifolia J.A. Schmidt in Mart. Fl. Bras. 8: 259. 1864. Localidade tipo: Brasil austral. São Paulo: Ilha do Cardoso, Prance 6933 (2.II.1978) MBM, Restinga.

Brunfelsia hydrangeaeformis (Pohl) Benth. ssp.hydrangeaeformis in DC. Prodr. 10: 198. 1846. Localidade tipo: Rio de Janeiro, prox. rio Paraybuna. Rio de Janeiro: Serra de Friburgo, Duarte 8445 (X.1964) HB, floresta.
Brunfelsia hydrangeaeformis ssp. capitata (Benth.) Plowman, Field Mus. Nat. Hist. Chicago Bot. n.s. 39:66.1998. Localidade tipo: Rio de Janeiro, Serra dos Órgãos. Minas Gerais: Coronel Pacheco, Heininger 460, RB; Espírito Santo: Venda Nova, Hatschbach 49870 (17.X.1981), MBM; Rio de Janeiro: Serra dos Orgãos, Brunet s/n (1888) R, floresta; São Paulo: Serra Bocaina, Lutz 14, R, floresta.

Brunfelsia latifolia (Pohl) Benth. in DC. Prodr. 10: 199. 1846. Localidade tipo: Rio de Janeiro. Rio de Janeiro: Pedra de Itauna, Sucre 5314 (06.IX.1969) RB, Restinga.

Brunfelsia obovata Benth. ssp. obovata in DC. Prodr. 10: 199. 1846. Localidade tipo: MG, Parahybuna. Minas Gerais: Carangola, Serra da Yarama, Kuhlmann 141 (19.IV.1935) RB.

Brunfelsia pauciflora (Cham. \& Schlecht.) Benth. ssp. pauciflora in DC. Prodr. 10: 199. 1846. Localidade tipo: Brasil meridional. Rio de Janeiro: Santa M. Madalena, Serra do Mocotó, Santos Lima s/n (VIII.1940) RB, floresta; São Paulo: Iguape, Morro das Pedras, Brade 8088 (IX.1920) RB.

Brunfelsia pilosa Plowman, Bot. Mus. Leafl. 24(2): 42. pl. XIV. 1974. Localidade tipo: Santa Catarina, São Miguel do Oeste. São Paulo: Carandirú, Tamandaré 244 (XII.1912) RB.

Brunfelsia rupestris Plowman, Fieldiana Bot. n.s. 8:7. Publ. 1322. 1981. Localidade tipo: Minas Gerais. Minas Gerais: Diamantina, Mello Barreto 9479 RB, campo rupestre.

Brunfelsia uniflora (Pohl) D. Don, Edinburgh New Philos. Journ. 86. 1829. Localidade tipo: Rio de Janeiro, Paraibuna. Minas Gerais: Viçosa, Kuhlmann s/n (19.XI.1928) RB; Espírito Santo: Goitacazes, Kuhlmann (10.XI.1943) RB; Rio de Janeiro: Porciúncula, Carauta et al. 4546 (I.1984) RB; São Paulo: Jacupiranga, Pabst \& Pereira 6023 (15.X.1961) HB. 
Diversidade taxonômica e distribuição geográfica das solanáceas que ocorrem no Sudeste

Brasileiro (Acnistus, Athenaea, Aureliana, Brunfelsia e Cyphomandra)

Cyphomandra Mart. ex Sendtn.

Cyphomandra calycina Sendtn., Flora 28: 167. tab. 1, fig. 1-7. 1845. Localidade tipo: Brasil. Minas Gerais: Viçosa, A. Soares (8.XII.1987) MBM; Rio de Janeiro: Munic. Paraty, M. Nadruz 469 et al. (15.XII.1988) $\mathrm{RB}$, floresta.

Cyphomandra diploconos (Mart.) Sendtn., Flora 28:169. tab. 3, fig. 1-6. 1845. Localidade tipo: Rio de Janeiro, Tijuca. Espírito Santo: Vitória, Pirani et al. 181 (s.d.) SP; Rio de Janeiro: Sumaré, Markgraf 31 (22.XI.1938) RB, floresta; São Paulo: P.E. Fontes do Ipiranga, Barros 596 (17.XII.1980) SP, floresta.

Cyphomandra divaricata (Mart.) Sendtn., Flora 28: 174. tab. 9, fig. 1-6. 1845. Localidade tipo: São Paulo, Lorena. Minas Gerais: Viçosa, Kuhlmann 1769 (03.II.1934) RB; Espírito Santo: Vitória, R.B. Mestre Alvaro, Pirani et al. 185 (21.XI.1982) SP; Rio de Janeiro: Petrópolis, Quitandinha, Otávio 68 (1948) SP, floresta; São Paulo: P.E. Fontes do Ipiranga, Handro s/n (23.XI.1932) SP, floresta.

Cyphomandra heterophylla Taub., Bot. Jahrb. Syst. 15, Beibl. 38: 16. 1893. Localidade tipo: Rio de Janeiro, São Cristovão. Rio de Janeiro: São Cristovão, Glaziou s/n. (27.IX.1880) P; São Paulo: Ilha da Queimada Grande, Hatschbach 4661 (03.XI.1920) SP.

Cyphomandra ovum-fringillae Dun. in DC. Prodr. 13(1): 394. 1852. Localidade tipo: Brasil: Bahia, Rio São Francisco, Serra Acuruá (Assuruá), 1838-1839. Rio de Janeiro: Estado da Guanabara, Serra do Medanha, D. Sucre 6405 et al. (27.XI.1969) RB, floresta; São Paulo: Campos Jordão, G. Hashimoto 23 (22.X.1938) RB, floresta.

Cyphomandra pinetorum Smith \& Downs, Phytologia 10: 436. tab. 9, fig. 8-10. 1964; Smith \& Downs, Fl. Ilustr. Catar. pp. 207-208. fig. 28, c-e. 1966. Localidade tipo: Santa Catarina,
Campo Alegre. Minas Gerais: Serra da Mantiqueira, Kuhlmann 2927 (06.XI.1953) SP; Rio de Janeiro: P.N. Serra do Itatiaia, Markgraf 3749 (28.XI.1938) SP, floresta; São Paulo: Campos do Jordão, Kuhlmann 2168 (25.XI.1949) SP, floresta.

Cyphomandra premnifolia (Miers) Dun., DC. Prodr. 13(1): 392. 1852. Localidade tipo: São Paulo. Rio de Janeiro: Serra do Mendanha, Martinelli et al. 4138 (04.IV.1978) $\mathrm{RB}$, floresta.

Cyphomandra sciadostylis Sendtn., Flora 28: 170. tab. 4, fig. 1-10. 1845. Localidade tipo: São Paulo, Mogi-Guaçu. Minas Gerais: Lavras, Leitão et al. 11815 (09.XII.1980) MBM; São Paulo: Serra da Cantareira, P. Doll s/n (19.X.1901) SP; Rio de Janeiro: Itatiaia, G. Hatschbach 35852 et al. 126. (1975) MBM.

Cyphomandra sycocarpa (Mart. \& Sendtn.) Sendtn. in Martius Fl. Bras. 10: 113. tab. 15. 1846. Localidade tipo: Bahia, Ilheus. Espírito Santo: Vitória, Kuhlmann 03 (20.III.1934) SP; Rio de Janeiro: Cabo Frio, Sucre et al. 9542 (23.VIII.1972) RB.

\section{RESULTADOS}

\section{ASPECTOS DO HÁBITO E HABITAT}

Os cinco gêneros, Acnistus, Athenaea, Aureliana, Brunfelsia e Cyphomandra, apresentam hábito de arbustivo a arbóreo, sendo predominante o arbustivo. Athenaea martiana e Brunfelsia clandestina são árvores de pequeno porte.

Em Acnistus arborescens e entre outras espécies de Cyphomandra pode-se observar as duas formas de vida, arbustiva e arbórea.

O ambiente preferencial dos 39 táxons é a floresta (tabela 1), com cerca de $80 \%$ na floresta ombrófila densa (floresta pluvial atlântica), $11 \%$ na ombrofila submontana ou 
Tabela 1 - Aspectos do hábito e do habitat. $\mathrm{AB}=$ Arbusto; $\mathrm{AV}=$ Árvore; $\mathrm{FL}=$ Floresta (não definida pelo coletor); $\mathrm{AT}=$ Atlântica; $\mathrm{MO}=$ Montana; $\mathrm{GA}=$ Galeria; $\mathrm{AR}=$ Araucária; $\mathrm{SE}=$ Secundária; $\mathrm{CE}=$ Cerrado; $\mathrm{CA}=\mathrm{Caatinga}$; $\mathrm{CM}=$ Campo; $\mathrm{CR}=$ Campo Rupestre; RE = Restinga; OU = Outros.

\begin{tabular}{|c|c|c|c|c|c|c|c|c|c|c|c|c|c|c|}
\hline \multirow{3}{*}{ TÁXONS } & \multirow{2}{*}{\multicolumn{2}{|c|}{ HÁBITO }} & \multicolumn{12}{|c|}{ TIPOS DE VEGETAÇÃO } \\
\hline & & & \multicolumn{6}{|c|}{ FLORESTAS } & \multirow{2}{*}{ CE } & \multirow{2}{*}{ CA } & \multirow{2}{*}{ CM } & \multirow{2}{*}{ CR } & \multirow{2}{*}{ RE } & \multirow{2}{*}{ OU } \\
\hline & $A B$ & AV & FL & AT & MO & GA & AR & SE & & & & & & \\
\hline \multicolumn{15}{|c|}{ Acnistus arborescens } \\
\hline \multirow{2}{*}{\multicolumn{15}{|c|}{$\begin{array}{l}\text { Athenaea anonacea } \\
\text { A. cuspidata }\end{array}$}} \\
\hline & & & & & & & & & & & & & & \\
\hline \multicolumn{15}{|l|}{ A. martiana } \\
\hline \multirow{2}{*}{\multicolumn{15}{|c|}{$\begin{array}{l}\text { A. micrantha } \\
\text { A. pereirae }\end{array}$}} \\
\hline & & & & & & & & & & & & & & \\
\hline \multirow{2}{*}{\multicolumn{15}{|c|}{$\begin{array}{l}\text { A. picta } \\
\text { A. pogogena }\end{array}$}} \\
\hline & & & & & & & & & & & & & \multicolumn{2}{|c|}{ A. pogogena } \\
\hline \multirow{2}{*}{\multicolumn{15}{|c|}{$\begin{array}{l}\text { Aureliana brasiliana } \\
\text { A. darcvi }\end{array}$}} \\
\hline & & & & & & & & & & & & & & \\
\hline \multicolumn{15}{|c|}{ A. fasciculata } \\
\hline \multirow{2}{*}{\multicolumn{15}{|c|}{$\begin{array}{l}\text { A. fasciculata var. longifolia } \\
\text { A. fasciculata var. tomentella }\end{array}$}} \\
\hline & & & & & & & & & & & & & & \\
\hline \multirow{2}{*}{\multicolumn{15}{|c|}{ A. tomentosa }} \\
\hline \multirow{2}{*}{\multicolumn{15}{|c|}{$\begin{array}{l}\text { A. velutina } \\
\text { A. wettsteiniana }\end{array}$}} \\
\hline & & & & & & & & & & & & & & \\
\hline \multicolumn{15}{|c|}{ Brunfelsia bonodora } \\
\hline \multicolumn{15}{|c|}{ B. brasiliensis } \\
\hline B. brasiliensis & & & & & & & & & & & & & & \\
\hline B. clandestina & & & & & & & & & & & & & & \\
\hline B. cuneifolia & & & & & & & & & & & & & & \\
\hline B. hydrangeae & & & & & & & & & & & & & & \\
\hline B. hydrangeae & & & & & & & & & & & & & & \\
\hline B. latifolia & & & & & & & & & & & & & & \\
\hline B. obovata & & & & & & & & & & & & & & \\
\hline $\begin{array}{l}\text { B. pauciflora } \\
\text { B. pilosa }\end{array}$ & & & & & & & & & & & & & & \\
\hline B. rupestris & & & & & & & & & & & & & & \\
\hline B. uniflora & & & & & & & & & & & & & & \\
\hline Cyphomandra & & & & & & & & & & & & & & \\
\hline C. diploconos & & & & & & & & & & & & & & \\
\hline C. divaricata & & & & & & & & & & & & & & \\
\hline C. heterophylls & & & & & & & & & & & & & & \\
\hline C. ovum-fringi & & & & & & & & & & & & & & \\
\hline $\begin{array}{l}\text { C. pinetorum } \\
\text { C. premnifolia }\end{array}$ & & & & & & & & & & & & & & \\
\hline $\begin{array}{l}\text { C. sciadostylis } \\
\text { C. sycocarpa }\end{array}$ & & & & & & & & & & & & & & \\
\hline
\end{tabular}

montana, $10 \%$ na ombrófila mista (floresta de araucaria), e $5 \%$ em estacional semidecidual (floresta de galeria).

A Brunfelsia cuneifolia, $B$. pauciflora, B. pilosa, Cyphomandra diploconos, $C$. divaricata e $C$. pinetorum foram coletadas em floresta de araucaria, mas também se desenvolvem na floresta atlântica. Como representante na floresta de galeria pode-se citar Aureliana velutina, Brunfelsia brasiliensis e B. cuneifolia. Observou-se ainda, que $25 \%$ das espécies coletadas em floresta secundária estão relacionadas entre as que ocorrem na floresta pluvial atlântica.

No brejo de altitude, floresta ombrófila densa montana, situado no Estado de Pernambuco (Sales et al. 1998) foram encontrados Acnistus arborescens e Brunfelsia uniflora.

A restinga, vegetação com influência marinha, está representada por cerca de $28 \%$ dos táxons (tabela 1 ).

A presença de Brunfelsia brasiliensis ssp. macrocalyx, $B$. clandestina, $B$. obovata, B. uniflora e Cyphomandra ovum-fringillae em cerrado, Brunfelsia brasiliensis em caatinga e B. rupestris em campo rupestre, indicam uma capacidade de adaptação dessas plantas, bem como a participação das solanáceas nessas formações vegetais. 


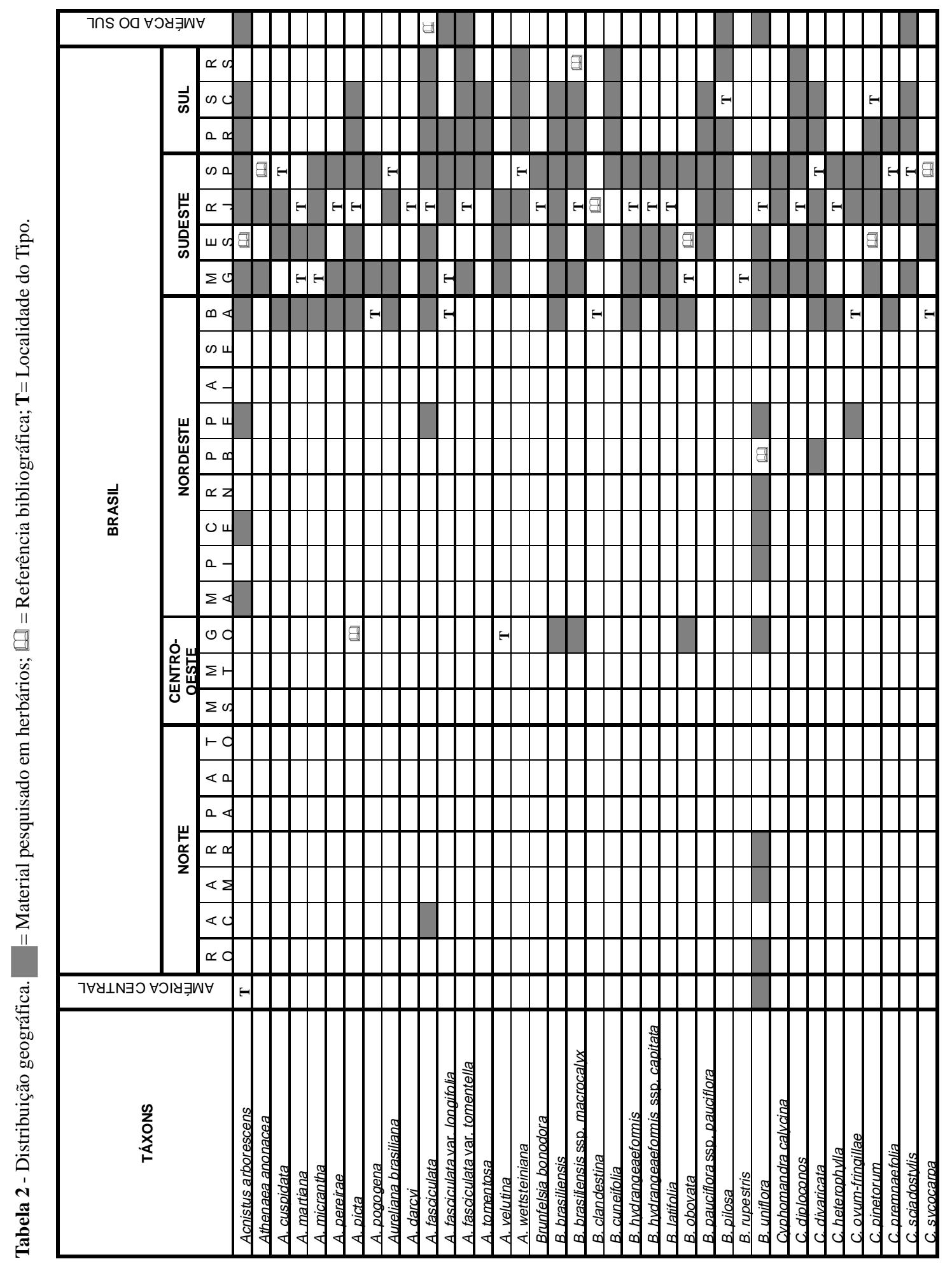


Representantes cosmopolitas como Acnistus arborescens (Rojas, 1997) e Brunfelsia uniflora (Plowmann, 1998) também foram encontrados nos mais diversos ambientes.

A presença de $12 \%$ dos táxons em "outros tipos de ambiente" (por exemplo, campos, alagados, brejos e capoeiras), não foram indicados devido a falta de clareza para a interpretação do ecossistema original, evidenciando também, a pouca precisão na informação sobre o local de coleta da planta.

\section{DISTRIBUIÇÃO GEOGRÁFICA}

As espécies que apresentam endemismo são Aureliana darcyi, localizada no estado do Rio de Janeiro e Aureliana angustifolia e Brunfelsia rupestris em Minas Gerais.

Tabela 3 - Táxons documentados em Unidades de Conservação. RB = Reserva Biológica; EB = Estação Biológica; $\mathrm{PE}=$ Parque Estadual; $\mathrm{APA}=$ Área de Proteção Ambiental; RF = Reserva Florestal; EE = Estação Ecológica; $\mathrm{PN}=\mathrm{Parque}$ Nacional. Unidades de Conservação segundo Buck, Viana Freire e Lima (1995).

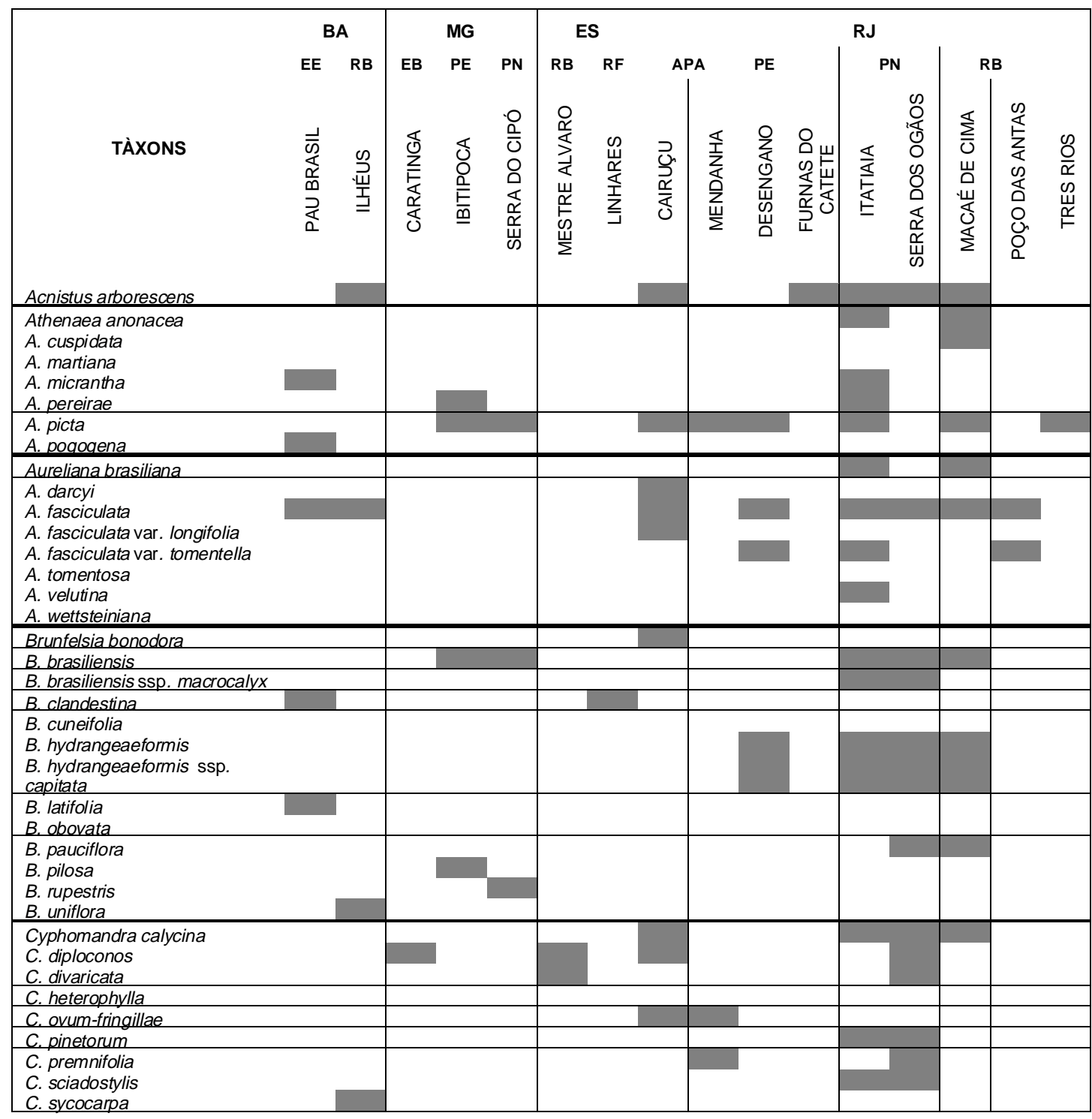


Diversidade taxonômica e distribuição geográfica das solanáceas que ocorrem no Sudeste Brasileiro (Acnistus, Athenaea, Aureliana, Brunfelsia e Cyphomandra)

A análise da distribuição geográfica dos táxons, demonstra alguma exclusividade para a região sudeste, documentada por Athenaea anonacea, Brunfelsia bonodora, Brunfelsia hydrangeaeformis ssp. capitata e Cyphomandra calycina, num total de $22 \%$ e extendendo-se para a região sul Athenaea picta, Aureliana fasciculata, A. tomentosa, $A$. wettsteiniana, Brunfelsia brasiliensis, $B$. cuneifolia, B. pauciflora, B. pilosa,
Cyphomandra diploconos, C. divaricata, $C$. pinetorum, $C$. premnifolia $e$. sciadostylis estimada em $19 \%$.

Pode-se citar dezenove espécies (Acnistus arborescens, Athenaea cuspidata, A. martiana, A. micrantha, A. pereirae, A. picta, A. pogogena, Aureliana brasiliana, A. fasciculata ( e var. longifolia), Brunfelsia brasiliensis, $B$. clandestina, B. hydrangeaeformis, $B$. latifolia, B. uniflora, Cyphomandra

Continuação da tabela 3 - Táxons documentados em Unidades de Conservação. RB = Reserva Biológica; EB = Estação Biológica; PE = Parque Estadual; APA = Área de Proteção Ambiental; RF = Reserva Florestal; EE = Estação Ecológica; PN = Parque Nacional. Unidades de Conservação segundo Buck, Viana Freire e Lima (1995).

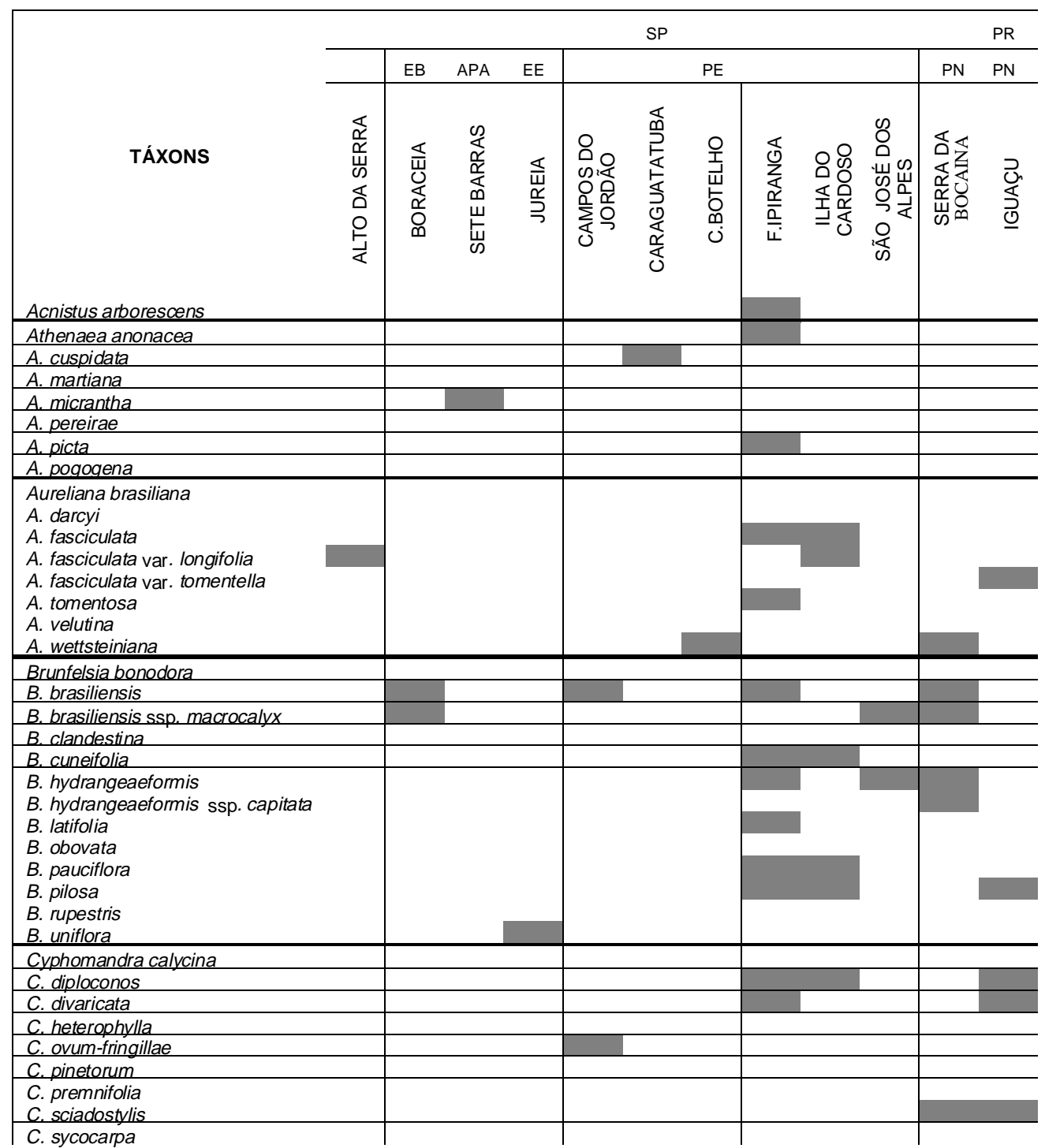


divaricata, C. heterophylla, C. ovumfringillae, C. premnifolia, C. sciadostylis e C. sycocarpa, provavelmente foram coletadas na floresta pluvial atlântica da regiãosul da Bahia (tabelas 1 e 2).

Duas espécies do gênero Cyphomandra ( $C$. divaricata $e$ C. premnifolia), representando $5 \%$ das espécies da região sudeste, ocupam a faixa florestal do estado da Bahia até o Rio Grande do Sul.

As espécies dos gêneros Athenaea e Brunfelsia, com exceção de B. uniflora e $B$. pilosa são exclusivamente brasileiras.

Seis táxons, Acnistus arborescens, as variedades de Aureliana fasciculata (var. longifolia e var. tomentella), Brunfelsia pilosa, Brunfelsia uniflora e Cyphomandra sciadostylis podem ser encontradas em outros países da América do Sul.

\section{ESPÉCIES EM UNIDADES DE CONSERVAÇÃO AMBIENTAL}

Dos cinco gêneros estudados, todas as espécies dos gêneros Acnistus e Aureliana foram localizadas crescendo em mais de uma unidade de conservação do país, (tabela $3)$.

Dos 39 táxons, somente três (03) ainda não foram localizados em unidade de conservação e são os relacionados a seguir: Athenaea martiana, Brunfelsia obovata e Cyphomandra heterophylla.

A quase totalidade das unidades de conservação (tabela 3), onde se localizam os táxons estudados, resguardam fragmentos e/ou remanescentes da floresta atlântica.

\section{“CHAVE PARA DETERMINAR OS TÁXONS, BASEADA NAS MONOGRAFIAS GENÉRICAS"}

1. Flores fasciculadas, longo pedunculadas (Acnistus)

A. arborescens

1. Flores não fasciculadas, sésseis ou pedunculadas; inflorescência de diversos tipos.

2. Estames com anteras de deiscência porícida, conectivo expandido 3. (Cyphomandra)

3. Estilete cilíndrico, por vezes um tanto dilatado no ápice, estigma geralmente menor que $2 \mathrm{~mm}$ de diâmetro.

4. Ovário e fruto com indumento de pubescente a piloso, tricomas com $1 \mathrm{~mm}$ de comprimento ou mais; conectivo pouco desenvolvido entre as tecas das anteras

C. pinetorum

4. Ovário e fruto glabros; conectivo bastante desenvolvido na região basal e entre as tecas das anteras.

5. Folhas inteiras e composto-ternadas; inflorescência menor que $5 \mathrm{~mm}$ de comprimento; tecas 3-4 mm de comprimento; estilete cilíndrico com ca. $5 \mathrm{~cm}$ de comprimento C. ovum-fringillae

5. Folhas inteiras; inflorescência maior que 5(até 30) cm de comprimento; tecas com mais de $7 \mathrm{~mm}$ de comprimento; estilete cilíndrico levemente dilatado no ápice com mais de $6 \mathrm{~cm}$ de comprimento C. divaricata

3. Estilete cilíndrico ou obcônico com ápice nitidamente ampliado, estigma geralmente maior que $2 \mathrm{~mm}$ de diâmetro, quando menor, maior que duas vezes o diâmetro da base do estilete. 
6. Conectivo fortemente expandido e ultrapassando as tecas na região basal ou apical das anteras.

7. Folhas e caules glabros; folhas inteiras e lobadas

C. sycocarpa

7. Folhas e caules pubescentes; folhas inteiras e pinatipartidas com 5 - 11 pinas .......

C. heterophylla

6. Conectivo mais espessado apenas na região basal das tecas das anteras.

8. Folhas de inteiras a pinatipartidas; estilete cilíndrico, estigma ampliado, obcônico ou claviforme.

9. Planta com folhas inteiras, pinatilobadas e pinatipartidas (5 - 9 pinas); estilete densamente pubescente, estigma ampliado ......................... C. sciadostylis

9. Planta com folhas inteiras, estilete glabro, estigma claviforme ...C. preminifolia

8. Folhas sempre inteiras; estilete infundibuliforme, estigma claviforme.

10. Lacínias do cálice quase do mesmo tamanho da corola ....................C. calycina

10. Lacínias do cálice menor que a metade do tamanho da corola .......C. diploconos

2. Estames com anteras de deiscência rimosa, conectivo não expandido.

11. Corola hipocraterimorfa; 5-estames, 2 pares desiguais; estigma reflexo e bífido; fruto capsular, sementes oblongas

(Brunfelsia)

12. Flores isoladas.

13. Flores alvas

B. clandestina

13. Flores violáceas tornando-se alvas com a idade

B. uniflora

12. Flores em inflorescências diversas.

14. Cálice 5-anguloso, plicado quando sêco.

15. Folhas cuneadas; pedicelo e cálice glandular, pubescente ou viloso, lacínias do cálice lanceoladas, com 3-7 mm comprimento; até 3 flores .... B. cuneifolia

15. Folhas obovadas; pedicelo e cálice glabro, lacínias do cálice largo-lanceoladas, com 1-4 mm comprimento; até 10 flores

B. obovata

14. Cálice cilíndrico, inflado quando sêco.

16. Folhas geralmente com $16-31 \mathrm{~cm}$ comprimento, subespatulada até oblanceolada; mais de 10 flores congestas "capituliformes" no ápice do ramo; cápsula totalmente envolvida pelo cálice.

17. Cálice pubescente ferrugíneo, tricomas simples

\section{B. hydrangeaeformis ssp. hydrangeaeformis}

17. Cálice glabrescente, tricomas glandulares

B. hydrangeaeformis ssp. capitata

16. Folhas geralmente com 2-15 cm comprimento; até 10 flores em inflorescência laxa lateral ou apical; cálice envolvendo total ou parcialmente a cápsula.

18. Folhas coriáceas, com margens fortemente revolutas

B. rupestris

18. Sem esse conjunto.

19. Cálice com tricomas simples.

20. Inflorescência com 1-3 flores, pedicelo articulado na porção distal; tubo da corola com 25-32 mm de comprimento B. pilosa

20. Inflorescência com 8 ou mais flores, pedicelo articulado na porção mediana, tubo da corola com 17-22 mm de comprimento

B. brasiliensis ssp. brasiliensis

19. Cálice glabro ou com tricomas glandulares esparsos.

21. Até 8 flores, tubo da corola com $25-40 \mathrm{~mm}$ comprimento; cálice envolvendo totalmente a cápsula. 
22. Pedicelo articulado na porção mediana

B. brasiliensis ssp. macrocalyx

22. Pedicelo ausente ou quase

B. pauciflora

21. Até 20 flores, tubo da corola com 15-25 mm comprimento; cálice envolvendo parcialmente a cápsula.

23. Folhas subsésseis, ovadas a obovadas, levemente acuminadas

B. latifolia

23. Folhas pecioladas, estreito-elípticas até lanceolada acuminada

B. bonodora

11. Corola rotácea, rotáceo-campanulada ou campanulada; 5-estames iguais; estigma ereto inteiro ou levemente bilobado; fruto bacáceo, sementes aplanadas, uniformes ou quase. 24. Plantas geralmente glabrescentes, sem tricomas glandulares; corola com ou sem máculas esverdeadas; cálice frutífero não acrescente 25. (Aureliana) 25. Ramos com 3-4 projeções ou alas longitudinais.

26. Corola com nervuras e máculas acastanhadas

A. tomentosa

26. Corola com nervuras e máculas esverdeadas.

27. Ápice da lacínia do cálice longo-acuminada

A. darcyi

27. Ápice da lacínia do cálice agudo.

28. Plantas glabrescentes; folhas com $15-24 \mathrm{~cm}$ comprimento

A. fasciculata var. longifolia

28. Plantas glabrescentes a pubescentes; folhas até $12 \mathrm{~cm}$ comprimento

29. Ramos e folhas glabrescentes ..A. fasciculata var. fasciculata

29. Ramos e folhas tomentosas .... A. fasciculata var. tomentella

25. Ramos sem projeções ou alas longitudinais.

30. Cálice com lacínias iguais

A. brasiliana

30. Cálice com lacínias desiguais, 1-3 mais desenvolvidas que as demais.

31. Folhas subsésseis, lacínias estreito-elípticas; inflorescência pauciflora;

lacínias do cálice estreito-lanceoladas

A. wettsteiniana

31.Folhas pecioladas, lâminas foliares ovadas; inflorescência multiflora,

lacínias do cálice largo-lanceoladas

A. velutina

24. Plantas glabrescentes e pilosas, tricomas glandulares por vezes presentes; corola com máculas vinosas; cálice frutífero acrescente

32. (Athenaea)

32. Tricomas glandulares presentes; cálice com lacínias iguais.

33. Lacínias do cálice cordiforme infladas

A. cuspidata

33. Lacínias do cálice de lanceoladas a ovadas.

34. Flores com mais de $10 \mathrm{~mm}$ comprimento; frutos de glabros a glabrescentes

A. picta

34. Flores com até $8 \mathrm{~mm}$ comprimento; frutos pilosos.

35. Lacínias da corola com margem glabra

A. micrantha

35. Lacínias da corola com margem ciliada

A. pogogena

32. Tricomas glandulares ausente; cálice com lacínias desiguais.

36. Folhas sésseis ou subsésseis com base decurrente

A. martiana

36. Folhas pecioladas, base de aguda a levemente decurrente.

37. Plantas vilosas; lacínias do cálice ovadas, fruto piloso ... A. pereirae

37. Plantas hirsutas; lacínias do cálice estreito-lanceoladas; fruto glabro

A. anonacea 


\section{DISCUSSÃO E CONCLUSÕES}

As espécies dos cinco gêneros, Acnistus, Aureliana, Athenaea, Brunfelsia e Cyphomandra, apresentam o hábito arbustivo e/ou arbóreo, sendo predominante o arbustivo. O número expressivo de táxons arbustivos evidência uma possível participação na composição do sub-bosque na floresta pluvial atlântica conforme Andreata et al. (1997) observaram para várias angiospermas coletadas na Reserva Ecológica de Macaé de Cima, inclusive para as Solanáceas.

O ambiente preferêncial desse grupo de espécies é a floresta em seus diversos tipos, classificada para a região segundo o sistema de Veloso et al. (1991), como ombrófila densa sub-montana ou montana (floresta pluvial atlântica), mista (floresta de araucaria), e estacional semidecidual (floresta de galeria).

Quase todas as espécies se desenvolvem na floresta pluvial atlântica (32 espécies), destas, seis foram coletadas também em floresta de araucaria.

As espécies coletadas em floresta secundária evidênciam condições de adaptação a esse tipo de alteração e que provavelmente são remanescentes da floresta atlântica, por ainda serem encontrados nesse ambiente, ou identificados como recolonizadores de áreas degradadas segundo alguns estudos florísticos que citam representantes da família.

A restinga, vegetação com influência marinha, está representada por onze (11) táxons com representação nas florestas pluvial atlântica e de araucaria.

Até o presente pode-se afirmar que Brunfelsia obovata ocorre exclusivamente em Cerrado e Brunfelsia rupestris em campo rupestre. Na caatinga foi coletado Brunfelsia brasiliensis, embora com capacidade de se adaptar em outros tipos de vegetação.

Os táxons dos gêneros Acnistus, Aureliana, Athenaea, Brunfelsia e Cyphomandra, possuem grande plasticidade, adaptando-se aos mais diversos ambientes da região sudeste, desde os mais úmidos, na floresta pluvial atlântica até os mais áridos cerrados encontrados nos estados de Goiás e Minas Gerais.

Da análise dos dados obtidos sobre a distribuição geográfica desse grupo de gêneros resultou na confirmação dos 39 táxons concentrados na região (Carvalho et al., 2000) e acrescentou novas localidades ampliando a ocorrência até a região sul do país.

O gênero monotipico, Acnistus arborescens, documentado por Hunziker (1992) para o México, América Central, Antilhas, América do Sul e Brasil, neste estudo é consolidado para os estados do nordeste e da região Sudeste/Sul.

Athenaea, gênero formado por sete espécies (Barbosa et al., 1989), exclusivamente brasileiro, é encontrado na natureza desde o estado da Bahia até Santa Catarina.

Todas as sete espécies de Aureliana (Hunziker et al. 1991, Carvalho et al. $1995 \mathrm{e}$ Almeida-Lafetá, 2000) estão representadas principalmente na região Sudeste/Sul..

As Brunfelsia com vinte e um táxons citados para o território brasileiro (Plowmam, 1998), treze (13) são assinalados para região de estudo e adjacências. Plowmam (1998) considera o Brasil como um possível centro secundário de especiação do gênero.

Das dezenove espécies de Cyphomandra registradas para o Brasil (Bohs 1994), nove estão localizadas na região sudeste. Esta região é tratada por Bohs (1994) como um possível centro de diversidade de espécies desse gênero.

O endemismo é observado nas espécies do gênero Athenaea, que são exclusivas do território brasileiro e identificado emAureliana darcyi, Aureliana angustifolia e Brunfelsia rupestris que foram coletados nos estados do Rio de Janeiro e de Minas Gerais respectivamente.

A riqueza de espécies concentradas na região sudeste é significativa, considerandose o número total de espécies de cada gênero 
analisado e acrescentando-se o número de espécies exclusivas para a região.

Estudos recentes sobre florística (Lima e Guedes-Bruni, 1977) destacam as solanáceas, entre outras famílias de angiospermas da Reserva Ecológica de Macaé de Cima no Rio de Janeiro, segundo o aspecto de riqueza de espécies.

De acordo com os critérios da IUCN 32 táxons, podem ser considerados protegidos, pela distribuição geográfica documentada através das coleções recentes de herbário e por ser possível localiza-los em unidades de conservação.

Entretando, uma espécie desperta preocupação - Brunfelsia rupestre, restrita a campo rupestre, que pode ser classificada como vulnerável, pela beleza da planta e pelo tipo de habitat exposto ao turismo.

Nota dos autores: a revisão gráfica deste artigo coincidiu com a divulgação de mais uma espécie para a região: Aureliana angustifolia Almeida-Lafetá, motivo pelo qual não foi possível inseri-la nas tabelas; uma novidade, arbusto descrito tomando por base as plantas coletadas na Reserva Biológica Santa Candida - Minas Gerais (Almeida-Lafetá, 2000), com vegetação do tipo floresta ombrofila densa.

\section{AGRADECIMENTOS}

Aos herbários nacionais pelo acesso ao material botânico, imprescindível para a realização deste artigo, bem como pela gentileza no atendimento.

Ao CNPq, pelas bolsas, concedidas aos autores, necessárias ao desenvolvimento deste Projeto.

Ao Sr. Presidente, Sérgio Bruni, do Instituto de Pesquisas Jardim Botânico do Rio de Janeiro e ao Diretor do Herbarium Bradeanum, pela gentil acolhida.

\section{REFERÊNCIAS BIBLIOGRÁFICAS}

Almeida-Lafetá, R. de C. 2000. A new species of Aureliana (Solanaceae) from Minas Gerais, Brazil. Novon 10(3):187-189, fig. 1A-M.

Andreata, R. H. P., Gomes, M., Baumgratz, J. F. A. 1997. Plantas herbáceo-arbustivas terrestres da Reserva Ecológica de Macaé de Cima. In: Lima, H. C. \& Guedes-bruni, R. R. (eds.). Serra de Macaé de Cima: Diversidade Florística e Conservação em Mata Atlântica. Rio de Janeiro, Instituto de Pesquisas Jardim Botânico do Rio de Janeiro, p. 65-73.

Barboza, G. E. \& Hunziker, A. T. 1989. Estudios sobre Solanaceae XXIX. Sinopsis taxonomia de Athenaea. Bol. Soc. Argent. Bot. 26(1-2):91-105, 6 figs.

Bohs, L. 1994. Cyphomandra (Solanaceae). Flora Neotropica. Monograph 63:1-175, 86 figs.

Bohs, L. 1995. Transfer of Cyphomandra (Solanaceae) end its species to Solanum. Taxon 44(4):583-587.

Bruck, E. C.; Viana Freire, A. M. \& Lima, M. F. 1995. Unidades de conservação no Brasil (Cadastramento e Vegetação 19911994). Centro de Sensoriamento Remoto, MMA. 224p.

Carvalho, L. d'A. F. 1997a. Diversidade Taxonômica das Solanáceas no estado do Rio de Janeiro (Brasil) - I. Albertoa 4(19):245-260, ilustr.

Carvalho, L. d'A. F. 1997b. Diversidade Taxonômica das Solanáceas no estado do Rio de Janeiro (Brasil) - II. Lycianthes e Solanum. Albertoa 4(21):281-300, ilustr. Carvalho, L. d'A. F. \& Bovini, M. G. 1995. Aureliana darcyi, a New Species of Solanaceae from Brazil. Novon 5(3): 257258, 7 figs.

D'Arcy, W. G. 1991. The Solanaceae since 1976, with a Review of its Biogeography. In Solanaceae III Taxonomy, Chemistry, Evolution. (Eds. J.G. Hawkes, R.N. Lester, M.Nee end N. Estrada): 75-137, figs. 
Góes-Filho, L. 1992. Atlas Nacional do Brasil, 2 ${ }^{\mathrm{a}}$ ed., Diretoria de Geociências. Rio de Janeiro, IBGE, 198 p.

Holmgren, P. K., Holmgren, N. H. \& Barnet, L. C. 1990. Index Herbariorum Part: 1. The herbaria of the world, $8 \mathrm{a}$ ed. New York Botanical Garden, New York.

Hunziker, A. T. 1979. South American Solanaceae: a synoptic survey. In The Biology Taxonomy of the Solanaceae (Eds. J.G. Hawkes, R.N. Lester and A.D. Skelding). Linn. Soc. Symp. Sér. 7:4986, figs

Hunziker, A. T. 1982. Estudios sobre Solanaceae. XXII. Revision sinoptica de Acnistus. Kurtziana 5:8-102, 1 fig.

Hunziker \& Barboza, G. E. 1991. Estudios sobre Solanaceae. XXX. Revision de Aureliana. Darwiniana 30(1-4):95-113, 6 figs.

Lima, H. C., Guedes-Bruni, R. R. 1997. Plantas Arbóreas da Reserva Ecológica de Macaé de Cima. In: Lima, H. C. \& Guedes-Bruni, R. R. (eds.). Serra de Macaé de Cima: Diversidade Florística e Conservação em Mata Atlântica. Rio de Janeiro, Instituto de Pesquisas Jardim Botânico do Rio de Janeiro, p.53-64

Lima, H. C., Guedes-Bruni, R. R. 1997. Diversidade de plantas vasculares na Reserva Ecológica de Macaé de Cima. In: Lima, H. C., Guedes-Bruni, R. R. (eds.). Serra de Macaé de Cima: Diversidade Florística e Conservação em Mata Atlântica. Rio de Janeiro, Instituto de Pesquisas Jardim Botânico do Rio de Janeiro, p.29-39.

Plowmam, T. C. (S. Knapp e J. R. Press, Eds) 1998. A revision of the South American species of Brunfelsia (Solanaceae). Fieldiana, Bot. n.s. 39, 135p.

Purdie, R. W., Symon, D. E. \& Haegi, L. 1982. Solanaceae. Flora of Australia 29:1208, 52 figs.

Rojas, C. E. B. 1997. Diversidad de las Solanaceae en los Andes de Venezuela.

Acta Bot. Venez. 20(1):81-92.
Sales, M. F., Mayo, S. J. \& Rodal, M. J. N. 1998. Plantas vasculares das florestas serranas de Pernambuco. eds. Univ. Fed. Rural de Pernambuco, Royal Botanic Gardens - Kew, CNPq., 130pp.

Sendtner, O. 1846. Solanaceae. Flora Brasiliensis 10:1-228, 14 tab.

Veloso, H. P., Rangel-Filho, A. L. R. \& Lima, J. C. A. 1991. Classificação da vegetação brasileira, adaptada a um sistema universal. Rio de Janeiro, IBGE. Departamento de Recursos Naturais e Estudos Ambientais, 124p. 
